\title{
Erratum to: Geometric phase and entanglement for a single qubit interacting with deformed-states superposition
}

\author{
K. Berrada • S. Abdel-Khalek • \\ C. H. Raymond Ooi
}

Published online: 13 March 2013

(C) Springer Science+Business Media New York 2013

\section{Erratum to: Quantum Inf Process DOI 10.1007/s11128-012-0510-8}

In the original version of this paper, the third affiliation of the corresponding author (K. Berrada) was incorrect. The correct affiliation is 'Department of Physics, Al-Imam Mohammad Ibn Saud Islamic University (IMSIU), College of Science, Riyadh, Saudi Arabia'.

The online version of the original article can be found under doi:10.1007/s11128-012-0510-8.

\section{K. Berrada $(\bowtie)$}

The Abdus Salam International Centre for Theoretical Physics, Strada Costiera 11,

Miramare-Trieste, Italy

e-mail: alonsoberlam@yahoo.com

\section{K. Berrada}

Laboratoire de Physique Théorique, Faculté des Sciences, Université Mohammed V-Agdal, Av. Ibn Battouta, B.P. 1014, Agdal Rabat, Morocco

\section{K. Berrada}

Department of Physics, Al-Imam Mohammad Ibn Saud Islamic University (IMSIU), College of Science, Riyadh, Saudi Arabia

\section{S. Abdel-Khalek}

Mathematics Department, Faculty of Science, Sohag University, 82524 Sohag, Egypt

S. Abdel-Khalek

Mathematics Department, Faculty of Science, Taif University, Taif, Saudi Arabia

C. H. R. Ooi

Department of Physics, University of Malaya, 50603 Kuala Lumpur, Malaysia 